\title{
Compressão Óptica para Sistemas de Transmissão em Altas Taxas
}

\author{
L. Zão e R. Coelho
}

\begin{abstract}
Resumo-Este trabalho apresenta um método de compressão óptica para altas taxas de transmissão $(10 \mathrm{~Gb} / \mathrm{s}$ para $40 \mathrm{~Gb} / \mathrm{s})$. A técnica de compressão proposta apresenta baixo custo em termos de componentes de amplificação óptica. Nas simulações, foram considerados sinais com padrões Gaussianos e não-Gaussianos (fractais). Para avaliação do desempenho, foram examinadas as medidas de BER (Bit Error Rate), diagrama de olho, estimação dos parâmetros estatísticos e a distribuição dos sinais. Os resultados demonstraram que, para valores semelhantes da BER, as distribuições e os parâmetros estatísticos dos sinais não foram alteradas pela compressão.
\end{abstract}

Palavras-Chave-compressão óptica, amplificação óptica, BER, diagrama de olho

Abstract-This paper presents an optical compression method for high speed data transmission $(10 \mathrm{~Gb} / \mathrm{s}$ to $40 \mathrm{~Gb} / \mathrm{s})$. The proposed compression method presents low costs related to optical amplifiers. For the simulations, signals with Gaussian and non-Gaussian (fractals) patterns were used. For performance evaluation, the following measures were considered: the bit error rate (BER), the eye diagram, the estatistical parameters and the signals distributions. The results demonstrated that, for similar Bit Error Rate (BER) measures, the distributions and the statistical parameters of the signals were not changed by the compression. by the compression.

Keywords - optical communications, optical compression, optical amplification.

\section{INTRODUÇÃO}

Nos últimos anos, as pesquisas para desenvolvimento de sistemas com comutação no domínio óptico, receberam forte impulso [1] [2]. Os benefícios das altas taxas de transmissão disponíveis neste meio, foram os principais agentes deste avanço. Geralmente, os sinais são provenientes do meio eletrônico, e, portanto, gerados em baixas taxas. Logo, para aproveitar a grande largura de banda disponível no meio óptico, é necessário que estes sinais sejam comprimidos para ajuste a taxas mais elevadas. Com esta finalidade, algumas técnicas de compressão óptica [3] [4] [5] [6] [7] têm sido apresentadas na literatura.

$\mathrm{O}$ alto custo dos componentes ópticos, tais como os amplificadores, ainda é um grande desafio para as técnicas de compressão realizadas inteiramente no domínio óptico. Em [6], foi proposto e desenvolvido, um método de compressão óptica para taxa de $2,5 \mathrm{~Gb} / \mathrm{s}$. Os sinais foram gerados eletronicamente com modulação FSK (Frequency-Shift Keying), por meio de placas FPGA (Field Programmable Gate Array). O

Leonardo Zão e Rosângela Coelho, Departamento de Engenharia Elétrica, Laboratório de Comunicações e Sistemas Ópticos (LaRSO), Instituto Militar de Engenharia (IME), Rio de Janeiro, Brasil. E-mail: \{coelho,zao\} @ ime.eb.br. esquema proposto atingiu taxa de compressão de 16:1 (de 150 $\mathrm{Mb} / \mathrm{s}$ para 2,5 Gb/s) com interessante desempenho.

No presente trabalho, este método de compressão óptica é avaliado para altas taxas (de $10 \mathrm{~Gb} / \mathrm{s}$ para $40 \mathrm{~Gb} / \mathrm{s}$ ). Esta técnica apresenta menor custo de componentes de amplificação óptica, na comparação com outros métodos de compressão apresentados na literatura [4]. Para avaliação de desempenho, foi utilizado um simulador óptico (OPTSIM, da RSOFT). Os pulsos ópticos foram gerados por uma fonte laser DFB (Distributed-Feedback) com comprimento de onda de 1550,92 $\mathrm{nm}$, na taxa de repetição de $10 \mathrm{~Gb} / \mathrm{s}$. A técnica OOK (On-Off Keying) foi aplicada na modulação dos pulsos ópticos.

O estudo do desempenho dos diferentes métodos de compressão óptica são normalmente avaliados a partir da BER e da relação sinal-ruído (SNR - Signal-to-Noise Ratio). Para melhor avaliar a compressão óptica, é necessário também considerar os impactos causados nas diferentes distribuições ou padrões dos sinais, bem como sobre seus parâmetros estatísticos. Assim, neste trabalho foram avaliados: a BER, as distribuições dos sinais Gaussianos e não-Gaussianos, gerados e recuperados após a compressão, e suas respectivas características estatísticas.

As sequências não-Gaussianas ou fractais foram representadas por processos estocásticos baseados no movimento Browniano fracionário fBm (fractional Brownian motion) [8]. $\mathrm{O}$ processo $\mathrm{fBm}$ foi escolhido por ser capaz de representar os diferentes graus de dependência temporal dos sinais. Esta dependência temporal é representada pelo parâmetro de Hurst $(0<H<1)$. A dependência temporal de um processo estocástico pode ser definida como a taxa de decaimento da sua função auto-correlação $\rho(k)$, quando $k \rightarrow \infty$. De acordo com o grau de dependência temporal, um processo estocástico pode ser classificado como: antipersistente $(0<$ $H<\frac{1}{2}$ ); com dependência de curto alcance (SRD - Short Range Dependence) $\left(H=\frac{1}{2}\right)$; ou com dependência de longo alcance (LRD - Long Range Dependence) $\left(\frac{1}{2}<H<1\right)$. Um processo $\mathrm{fBm}$ é completamente definido por seus parâmetros média $(m)$, variância $\left(\sigma^{2}\right)$ e parâmetro de $\operatorname{Hurst}(H)$.

O restante do trabalho está organizado da seguinte forma: a seção II apresenta o método de compressão óptica avaliado. A seção III descreve o experimento realizado através de simulação óptica para avaliar o método de compressão óptica proposto neste trabalho. A seção IV mostra e discute os principais resultados da avaliação de desempenho da compressão. Finalmente, a seção V conclui o presente trabalho. 


\section{MÉTOdO DE COMPRESSÃo ÓPTICA}

O método de compressão óptica proposto neste trabalho é exemplificado na Fig. 1.

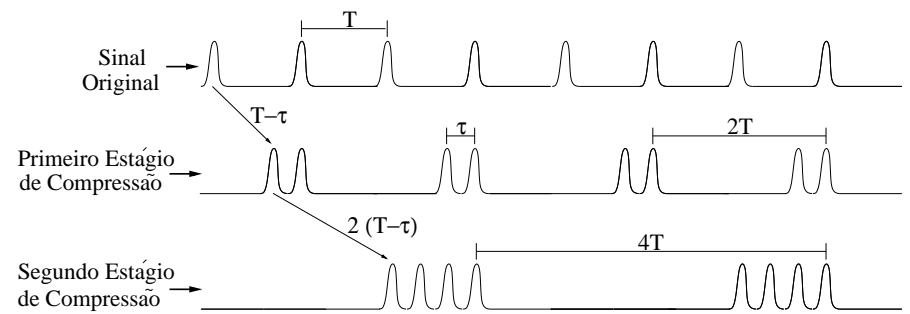

Fig. 1. Exemplificação do método de compressão óptica proposto.

A compressão é realizada em $n$ estágios consecutivos. Desta forma, grupos de $2^{n}$ bits (pulsos ópticos) são formados após a compressão óptica. A Fig. 2 mostra os componentes ópticos propostos para cada estágio de compressão.

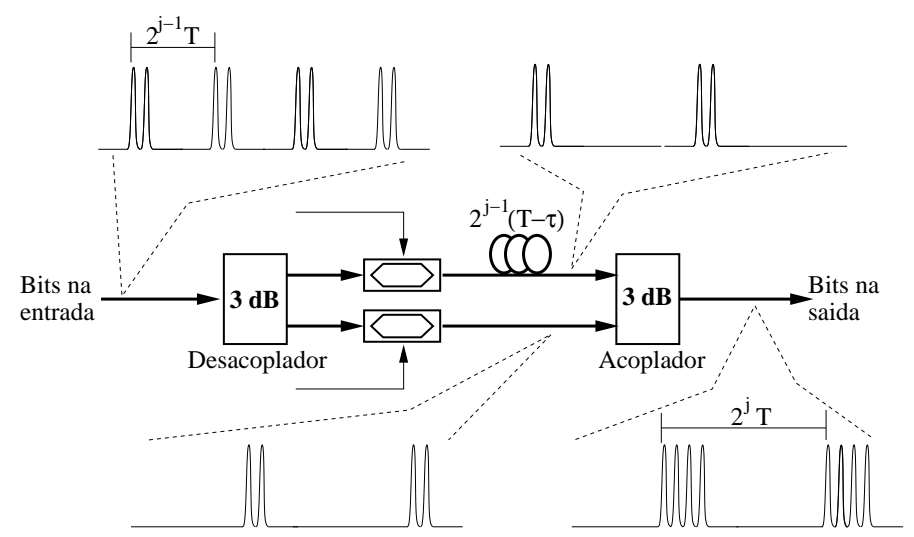

Fig. 2. j-ésimo estágio de compressão: pares de grupos de $2^{j-1}$ bits são comprimidos em um grupo de $2^{j}$ bits.

$\mathrm{Na}$ entrada de $j$-ésimo estágio de compressão, chegam grupos de $2^{j-1}$ bits, com intervalo $2^{j-1} T$ entre grupos, e $\tau$ entre bits do mesmo grupo. Utilizando-se de acopladores ópticos com 3dB de perdas, moduladores Mach-Zender (MZ) e linhas de retardo (FDL - Fiber Delay Lines), cada par de grupos de bits consecutivos são separados em fibras distintas, um destes grupos é atrasado de $2^{j-1}(T-\tau)$, e, em seguida, os grupos são re-acoplados na mesma fibra, formando um único grupo de $2^{j}$ bits.

Neste trabalho, foram utilizados nos experimentos 4 estágios de compressão, resultando em grupos de bits (pacotes) de tamanho $2^{4}=16$ bits. $\mathrm{O}$ sinal a ser comprimido é gerado à taxa de $f_{0}=10 \mathrm{~Gb} / \mathrm{s}$, e convertido para uma taxa de $f_{1}=40$ $\mathrm{Gb} / \mathrm{s}$. Assim,

$$
\begin{gathered}
T=\frac{1}{f_{0}}=100 \mathrm{ps} \\
\tau=\frac{1}{f_{1}}=25 \mathrm{ps}
\end{gathered}
$$

Uma grande vantagem do método de compressão apresentado é que ele não possui limitação quanto ao tamanho do grupo formado, ou quanto às taxas utilizadas. Contudo, a formação de grupos muito grandes (com mais de 4096 bits) irá requerer grande custo de componentes ópticos [3] [4].
$\mathrm{Na}$ maioria das soluções apresentadas na literatura [4], seriam necessários até 8 amplificadores ópticos para a compressão com 4 estágios. Na compressão proposta, como solução de baixo custo, um único amplificador óptico EDFA (Erbium Doped Fiber Amplifier) é utilizado, para compensar parte das atenuações sofridas pelo sinal nos diversos componentes ópticos. Contudo, quanto maior for o tamanho $b$ dos grupos formados, maior será o número $n$ de estágios de compressão necessários $\left(n>\log _{2} b\right)$. Consequentemente, um número maior de amplificadores deverão ser utilizados, como é o caso mostrado em [9].

\section{O EXPERIMENTO}

Nos experimentos foram realizadas diversas simulações para avaliação do método de compressão óptica. O simulador óptico utilizado foi o OPTSIM, da RSOFT, na versão Linux [10]. A Fig. 3 ilustra a simulação realizada para a avaliação do desempenho da compressão.

A largura dos pulsos ópticos é de 15 ps, com comprimento de onda de 1550,92 nm, e foram gerados à frequência de 10 GHz. Em seguida, estes pulsos são modulados por um sinal elétrico capaz de representar os padrões Gaussiano e fBm. A Fig. 4a mostra uma sequência do sinal óptico contendo 16 bits à taxa de $10 \mathrm{~Gb} / \mathrm{s}$ (intervalo $T=0,1 \mathrm{~ns}$ ), resultante da modulação OOK dos pulsos ópticos (antes da compressão).

Este sinal foi então comprimido através dos 4 estágios de compressão, formando grupos de 16 bits, ou seja, para a taxa de $40 \mathrm{~Gb} / \mathrm{s}$. Entre o segundo e o terceiro estágios de compressão, o sinal foi amplificado por um amplificador EDFA de $14 \mathrm{dBm}$ de potência. O propósito deste amplificador único é compensar as atenuações sofridas pelo sinal durante os estágios, minimizando o custo com componentes ópticos. A Fig 4b mostra a sequência do sinal óptico resultante da compressão dos 16 bits. Este sinal foi captado após o quarto estágio de compressão, à taxa de $40 \mathrm{~Gb} / \mathrm{s}$ (com intervalo $\tau=0,025$ ns entre bits).

Ao final do quarto estágio de compressão, é somado ao sinal óptico um ruído Gaussiano, como forma de compensar os componentes ideais da simulação (acopladores). A variação da potência deste ruído é utilizada como instrumento para avaliação do impacto da compressão sobre os diversos sinais, para diferentes valores da BER. Após a compressão óptica, o sinal óptico é convertido para o domínio eletrônico por um fotodetector PIN. Finalmente, este sinal elétrico é amostrado e quantizado através de um conversor A/D (analógico/digital), com frequência de amostragem de $40 \mathrm{GHz}$. A partir desta digitalização, os padrões e características estatísticas dos sinais foram recuperados e avaliados.

Nas simulações realizadas, foram utilizadas três sequências distintas: uma sequência Gaussiana (Gauss), uma sequência $f B m$ LRD com $H=0,8\left(f B m \_08\right)$ e uma sequência $f B m$ SRD com $H=0,5$ (fBm_05). Estas sequências foram escolhidas para representar diferentes características dos sinais quanto ao grau de dependência temporal. A Tab. I mostra os parâmetros média $(m)$, variância $\left(\sigma^{2}\right)$ e Hurst $(H)$ estimados para as três sequências. Cada uma das sequências amostrais é composta de $2^{13}=8096$ bits. O objetivo é examinar o impacto do método de compressão nos diferentes padrões de sinais e estatísticas. 


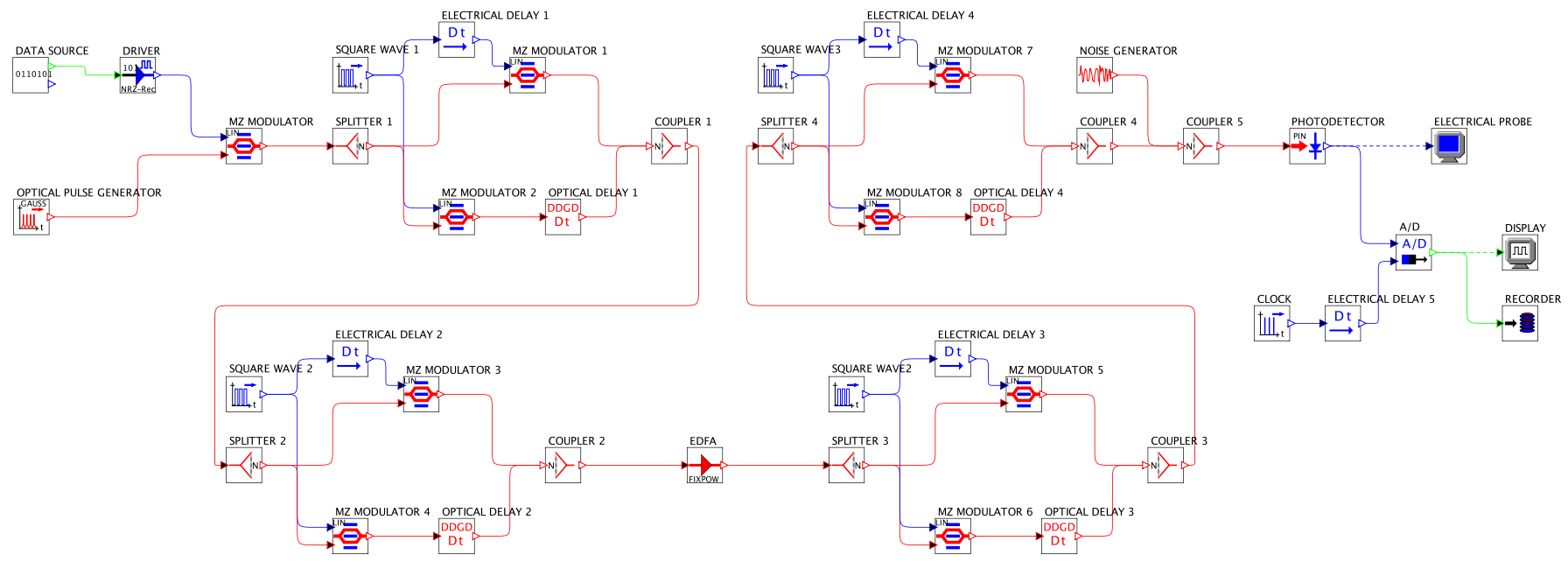

Fig. 3. Experimento: simulação da compressão de quatro estágios (OPTSIM/RSOFT).
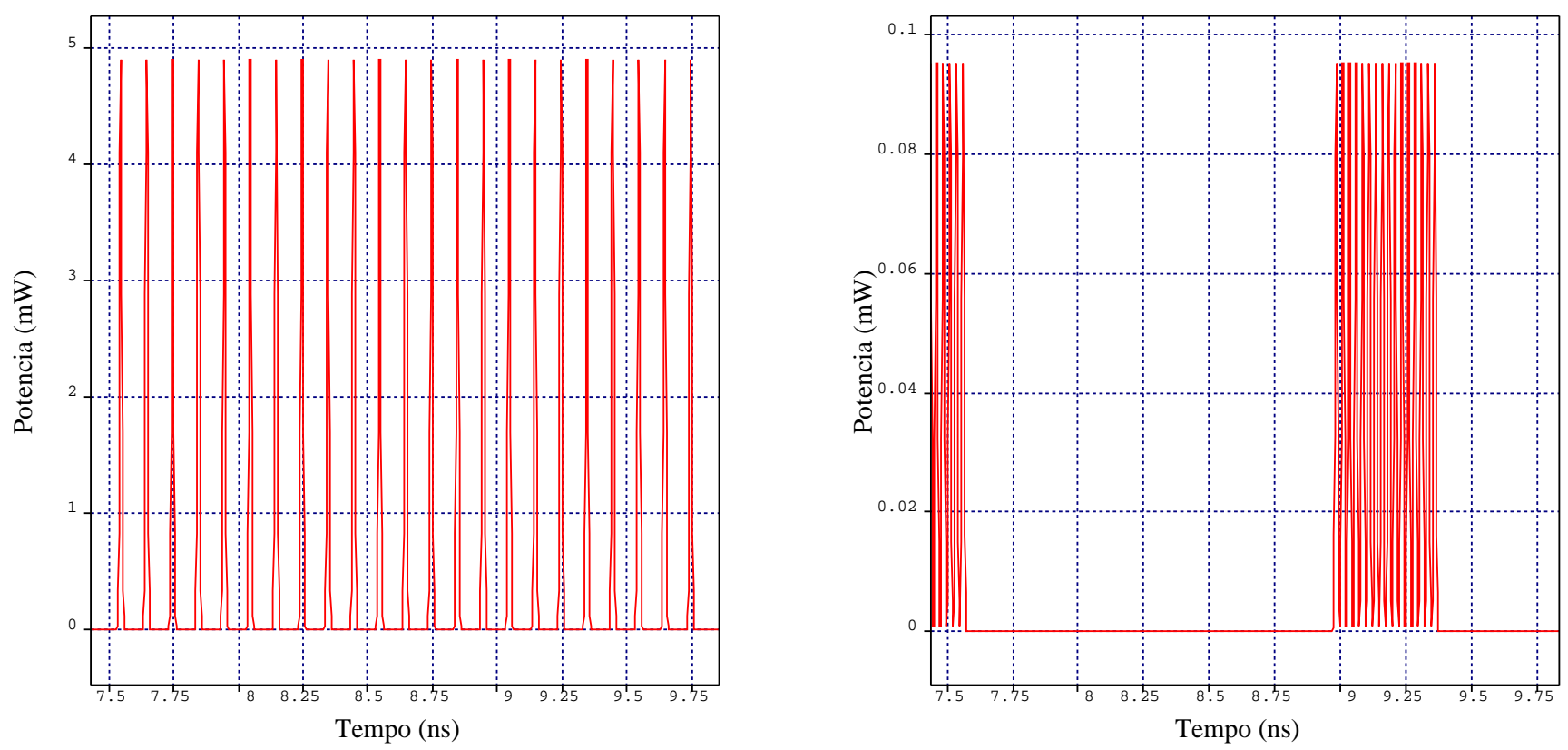

Fig. 4. a) Amostra de uma sequência de 16 bits antes da compressão e b) a sequência resultante da compressão óptica.

TABELA I

PARÂMETROS DAS SEQUÊNCIAS ANTES DA COMPRESS ÃO.

\begin{tabular}{|l|c|c|c|}
\hline Sequência & média $(m)$ & Variância $\left(\sigma^{2}\right)$ & Hurst $(H)$ \\
\hline Gauss & 19,39 & 19,30 & 0,46 \\
fBm_08 & 18,04 & 20,93 & 0,81 \\
fBm_05 & 19,16 & 21,24 & 0,58 \\
\hline
\end{tabular}

Nos experimentos, também foram realizadas as medidas de BER e o diagrama de olho. Todas as estimações dos parâmetros estatísticos das sequências consideradas neste trabalho foram realizadas com o software SET (Scaling Estimation Tool) [11]. Para a estimação do parâmetro de Hurst, o SET possui três diferentes métodos: R/S, Higuchi e Wavelets. Neste trabalho foi adotado o método de Higuchi [12].

\section{Resultados}

Para cada uma das sequências Gauss, fBm_08 e fBm_05, foram realizadas três simulações. Em cada simulação, ruídos Gaussianos com $-26 \mathrm{dBm},-24 \mathrm{dBm}$ e $-23 \mathrm{dBm}$ de potência foram acrescidos ao sinal no final da compressão. Estes níveis de ruído foram utilizados para alcançar medidas da BER na faixa de valores de $10^{-14}$ a $10^{-7}$.

A Tab. II mostra os parâmetros estatísticos média $(m)$, variância $\left(\sigma^{2}\right)$ e o parâmetro de Hurst $(H)$ para a sequência Gauss recuperada ao final da compressão óptica. Os valores das medidas da BER dos experimentos também são expressas na Tab. II.

Note que os parâmetros estatísticos da sequência Gauss original não sofreram alterações significativas, quando comparados aos valores da Tab. I. Isto pode ser observado para 
TABELA II

PARÂMETRos estatísticos E BER da SEQUÊNCIA Gauss RECUPERADA APÓS A COMPRESSÃO.

\begin{tabular}{|c|c|c|c|c|}
\hline Ruído & média $(m)$ & Variância $\left(\sigma^{2}\right)$ & Hurst $(H)$ & BER \\
\hline$-26 \mathrm{dBm}$ & 19,37 & 19,36 & 0,46 & $1,44 \times 10^{-13}$ \\
$-24 \mathrm{dBm}$ & 19,31 & 19,16 & 0,47 & $4,00 \times 10^{-10}$ \\
$-23 \mathrm{dBm}$ & 19,20 & 18,80 & 0,46 & $2,59 \times 10^{-7}$ \\
\hline
\end{tabular}

as três medidas de BER obtidas nos experimentos. Logo, o método de compressão proposto pode ser utilizado em sinais Gaussianos, resultando em diferentes níveis de BER, sem alterar suas principais características estatísticas.

Os resultados das simulações realizadas com as sequências fBm_08 e fBm_05 estão expressos nas Tabs. III e IV, respectivamente.

\section{TABELA III}

PARÂMETRos ESTATÍsticos E BER DA SEQUÊNCIA $f B m \_08$ RECUPERADA APÓS A COMPRESSÃO.

\begin{tabular}{|c|c|c|c|c|}
\hline Ruído & média $(m)$ & Variância $\left(\sigma^{2}\right)$ & Hurst $(H)$ & BER \\
\hline$-26 \mathrm{dBm}$ & 18,03 & 20,88 & 0,81 & $7,10 \times 10^{-14}$ \\
$-24 \mathrm{dBm}$ & 17,95 & 20,94 & 0,81 & $6,30 \times 10^{-10}$ \\
$-23 \mathrm{dBm}$ & 17,86 & 20,96 & 0,81 & $1,87 \times 10^{-7}$ \\
\hline
\end{tabular}

TABELA IV

PARÂMETRos ESTATÍSTICOS E BER DA SEQUÊNCIA $f B m \_05$ RECUPERADA APÓS A COMPRESSÃO.

\begin{tabular}{|c|c|c|c|c|}
\hline Ruído & média $(m)$ & Variância $\left(\sigma^{2}\right)$ & Hurst $(H)$ & BER \\
\hline$-26 \mathrm{dBm}$ & 19,14 & 21,18 & 0,58 & $1,09 \times 10^{-13}$ \\
$-24 \mathrm{dBm}$ & 19,06 & 21,15 & 0,59 & $6,83 \times 10^{-10}$ \\
$-23 \mathrm{dBm}$ & 18,97 & 21,20 & 0,59 & $1,19 \times 10^{-7}$ \\
\hline
\end{tabular}
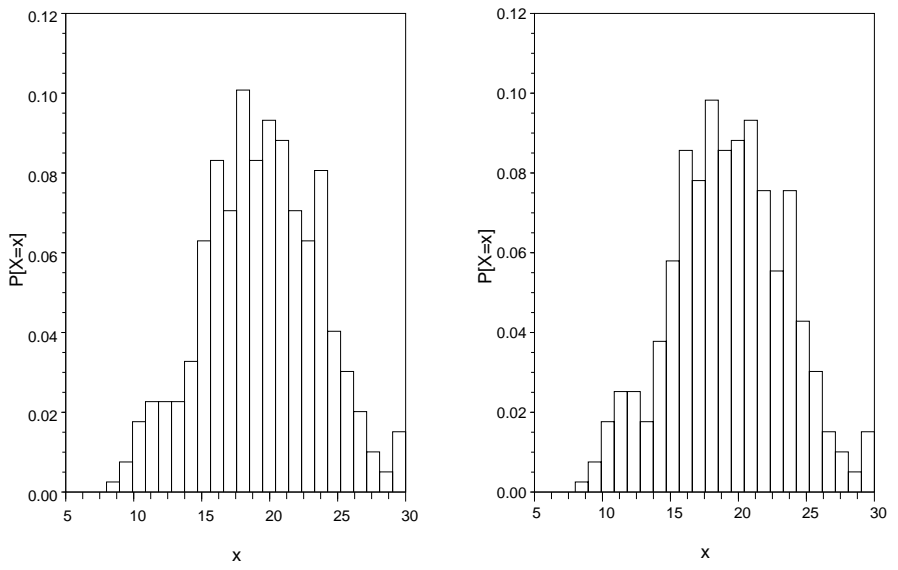

Fig. 5. a) fdp da sequência Gauss original e b) fdp da sequência Gauss recuperada após a compressão $\left(B E R=10^{-10}\right)$.

Pode-se observar que os sinais representados por processos estocásticos $\mathrm{fBm}$, também não sofreram alterações em seus parâmetros estatísticos. Isto ocorreu para os dois casos de dependência temporal estudados (SRD e LRD). Ou seja, estes resultados mostraram que, para valores semelhantes de
BER, a compressão óptica não afetou os parâmetros estatísticos dos sinais considerados. Isto demonstra que, para sinais Gaussianos e não-Gaussianos, e para diferentes graus de dependência temporal, o método de compressão proposto não altera as características estatísticas dos sinais.

A Fig. 5 mostra as funções densidade de probabilidade (fdp) das sequências Gauss original e recuperada após a compressão óptica, para um nível de ruído de $-24 \mathrm{dBm}$. As Figs. 6 e 7 mostram resultados análogos, para as sequências $f B m \_08$ e $f B m_{-} 05$, respectivamente. Este nível de ruído resultou, para as três sequências, em valores de BER da ordem de $10^{-10}$.
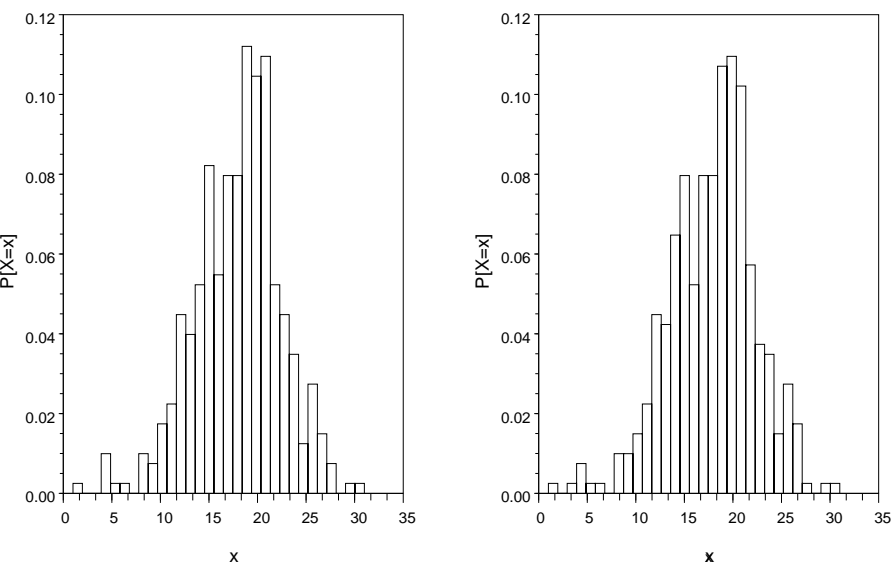

Fig. 6. a) fdp da sequência $f B m_{-} 08$ original e b) fdp da sequência $f B m_{-} 08$ recuperada após a compressão $\left(B E R=10^{-10}\right)$.
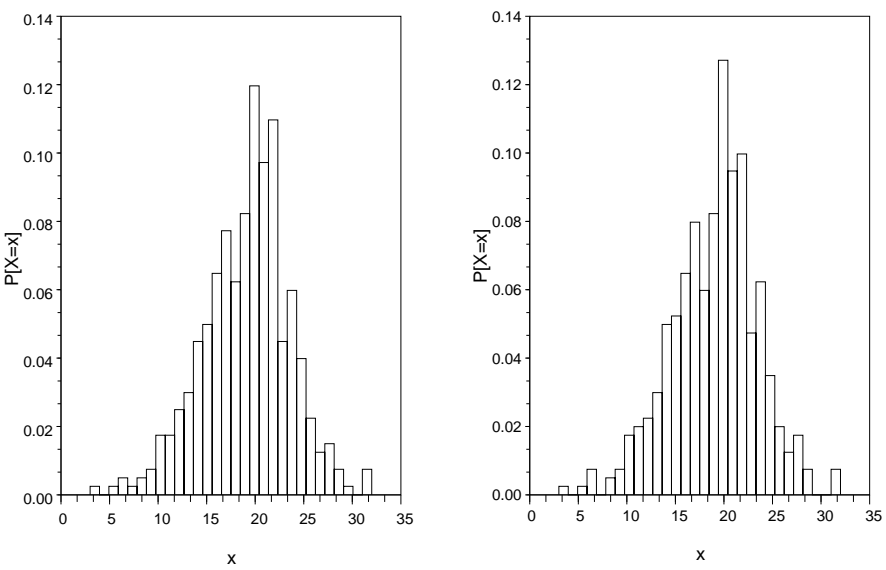

Fig. 7. a) fdp da sequência $f B m \_05$ original e b) fdp da sequência $f B m \_05$ recuperada após a compressão $\left(B E R=10^{-10}\right)$.

As Figs. 5, 6 e 7 mostram que, para as medidas de BER consideradas, os sinais não sofreram alterações em suas distribuições.

A Fig. 8 apresenta a variação da BER versus a potência do sinal óptico na entrada do primeiro estágio da compressão, para as três sequências amostrais.

Percebe-se que os diferentes padrões de sinais tiveram 


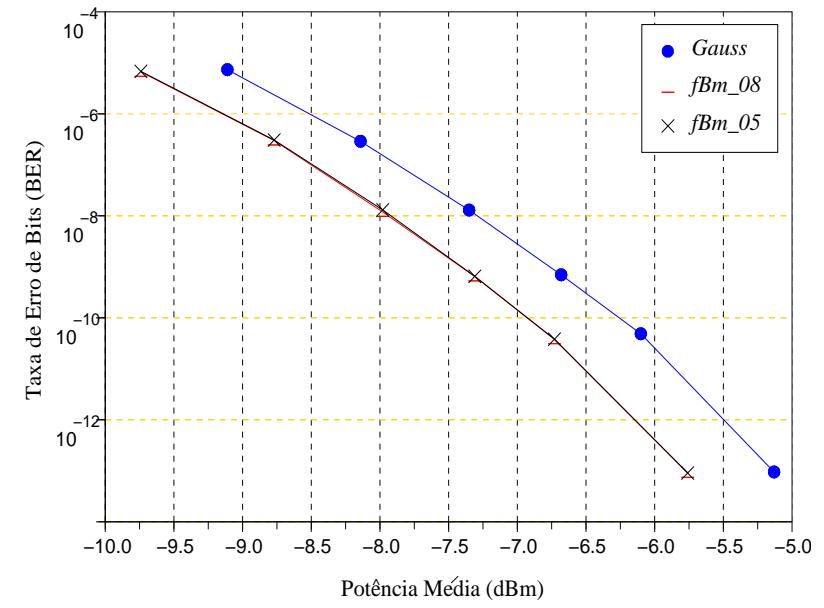

Fig. 8. BER medida dos sinais na saída do fotodetector, após a compressão.

comportamentos semelhantes com relação aos valores da BER para os diferentes níveis de potência do sinal.

A Fig. 9 mostra o diagrama de olho obtido na saída do fotodetector PIN, para o sinal gerado a partir da sequência Gauss.

\section{CONCLUSÃo}

Este trabalho apresentou um método de compressão óptica, baseado em 4 estágios de compressão consecutivos. No experimento aqui apresentado, a compressão é realizada a partir de um sinal de entrada com taxa de $10 \mathrm{~Gb} / \mathrm{s}$, resultando em um sinal comprimido com taxa de $40 \mathrm{~Gb} / \mathrm{s}$. Assim, uma taxa de compressão de 4:1 é obtida com esta proposta.

Uma das principais vantagens da compressão óptica apresentada neste trabalho é o seu baixo custo com componentes ópticos. Apenas um amplificador óptico é utilizado na compressão do sinal.

Para avaliação da compressão, foram utilizados sinais com distribuições Gaussiana (sequência Gauss) e fBm (sequências fBm_08 e fBm_05). Estes padrões são representantes de processos com dependências de longo e curto alcance. Como sinais com diferentes aplicações possuem geralmente padrões distintos, é de fundamental importância que o método de compressão proposta não cause impactos sobre diferentes padrões de sinais. Para que estes impactos da compressão óptica sobre os diferentes sinais pudessem ser avaliados, ruídos Gaussianos foram adicionados ao sinal durante a compressão. Desta forma, foram obtidas medidas da BER no intervalo de $10^{-14}$ a $10^{-7}$.

Os resultados mostraram que, para diferentes níveis de ruído, a compressão óptica não alterou os valores de média, variância e parâmetro de Hurst do sinais considerados. Além disto, não houve impacto significativo da compressão sobre as distribuições dos diferentes sinais, para valores de BER da ordem de $10^{-10}$.

Assim, o método de compressão óptica proposto conseguiu realizar a compressão para altas taxas, de sinais com diferentes distribuições e graus de dependência temporal, sem alterar seus padrões ou características estatísticas.

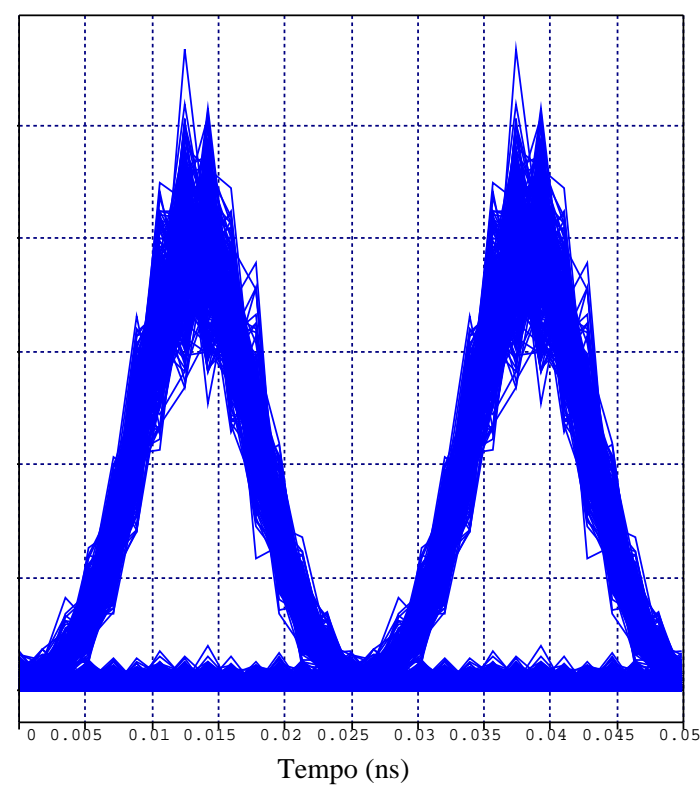

Fig. 9. Diagrama de olho do sinal elétrico na saída do fotodetector, para valor de $B E R=4,00 \times 10^{-10}$.

\section{REFERÊNCIAS}

[1] G. P. Agrawal, Fiber Optic Communication Systems. John Wiley \& Sons, Inc., 1951.

[2] R. Ramaswami and K. N. Sivarajan, Optical Networks, A Practical Perspective. USA: Morgan Kaufmann Publishers, 2 ed., 2002.

[3] P. Almeida et al, "All-optical packet compression based on time-towavelength conversion," IEEE Photonics Technology Letters, vol. 16, pp. 1688-1690, July 2004

[4] S. Seo, K. Bergman, and P. Prucnal, "Transparent optical networks with time-division multiplexing," IEEE JSAC, vol. 14, pp. 1039-1051, June 1996.

[5] S. Aleksic, "Packet-switched OTDM networks employing the packet compression/expansion technique," in Photonic Network Communications, pp. 273-288, kluwer Academic Publishers, 2003.

[6] L. Zão and R. Coelho, "Optical compression for samples with different bit patterns and scaling degrees," Optical Engineering, vol. 48, no. 3, pp. 035002-1-035002-5, 2009.

[7] H. P. S. Rangarajan and D. Blumenthal, "All-optical packet compression of variable length packets from 40 to $1500 \mathrm{~b}$ using a gated fiber loop," IEEE Photonics Technology Letters, 2006.

[8] B. Mandelbrot and J. van Ness, "Fractional brownian motions, fractional noises and applications," SIAM Review, 1968.

[9] L. Zão and R. Coelho, "Método de compressão híbrida para geração de pacotes Ópticos," Anais do XXII Simpósio Brasileiro de Telecomunicações, pp. 962-967, September 2005.

[10] RSOFT, "Optsim user manual," 2003. Artis Software Coroporation

[11] L. Zão, J. Filho, A. Pereira, M. Diniz, and R. Coelho, "SET (scaling estimation tool): Uma ferramenta gráfica de estimação e análise de sistemas com características de dependência temporal," XXIV Simpósio Brasileiro de Redes de Computadores, Maio 2006.

[12] T. Higuchi, "Approach to an irregular time series on the basis of the fractal theory," Phys. D, vol. 31, no. 2, pp. 277-283, 1988. 